\title{
Neuropsychiatric Symptoms in the Course of Parkinson's Disease
}

\author{
Kasia Gustaw Rothenberg* \\ Lou Ruvo Center for Brain Health, Neurological Institute, Cleveland Clinic Foundation, USA \\ *Corresponding author: Kasia Gustaw Rothenberg, Lou Ruvo Center for Brain Health, Neurological Institute, Cleveland \\ Clinic Foundation, USA
}

\section{ARTICLE INFO}

Received: 幽 July 03, 2020

Published: 幽 July 14, 2020

Citation: Kasia Gustaw Rothenberg. Neuropsychiatric Symptoms in the Course of Parkinson's Disease. Biomed J Sci \& Tech Res 28(5)-2020. BJSTR. MS.ID.004713.

Abbreviations: DLB: Dementia with Lewy Bodies; MCI: Mild Cognitive Impairment; AD: Alzheimer Disease; EDS: Excessive Daytime Sleepiness; EEG: Electroencephalography; GBA: Glucocerebrosidase; MAPT: Microtubule-Associated Protein Tau; COPT: Catechol-O-Methyl Transferase; APOE: Apolipoprotein E; DA: Dopamine Agonists; TCAS: Tricyclic Antidepressants; SSRIs: Selective Serotonin Reuptake Inhibitors; SNRIs: Serotonin Noradrenaline Reuptake Inhibitors; ECT: Electroconvulsive Therapy; TMS: Transcranial Magnetic Stimulation; EDS: Excessive Daytime Sleepiness; AAN: American Academy of Neurology; ICDS: Impulse Control Disorders; OCD: Obsessive Compulsive Disorder; DAWS: Dopamine Agonist Withdrawal Symptoms
ABSTRACT

Psychiatric symptoms associated with Parkinson's Disease (PD) are common and represent a special challenge because many of the psychiatric syndromes not only emerge in the process of neurodegeneration of Parkinson's disease, but could be a consequence of treatment of the underlying motor dysfunction. The spectrum of psychiatric symptoms associated with PD include: cognitive dysfunction and dementia, psychosis (especially hallucinations), mood disorders (depression and apathy), anxiety, sleep disturbances, fatigue. Mood disorders associated with PD require thorough assessment since some of the core symptoms of PD like bradykinesia or psychomotor slowness may be mistaken for affective component of primarily depressive disorders. Patient may benefit from carefully monitored regiment of antidepressants from various groups including dopamine agonists, TCA, SSRI Cognitive impairment is increasingly recognized as intrinsic features of PD process. Memory impairment is less prominent early on, while executive dysfunction and visual spatial impairments could present relatively early in the course of the process. PD (PDD) dementia frequently clusters with other neuropsychiatric symptoms including the most problematic one-psychosis. Some psychiatric symptoms, mainly: impulse control disorders, psychosis, irritability, agitation, dysphoria may be associated with treatment of motor symptoms of PD. The effectiveness of pharmacotherapy for those symptoms is questionable.

Psychosis seems to be one of the most cumbersome symptoms in PD since it can emerge as a neuropsychiatric symptom of the disease or/and the side effect of the treatment. Consensus clinical criteria have emerged for psychosis in PD. In terms of pharmacotherapy, clozapine has consistently shown clinical benefit. Quetiapine is widely prescribed for the treatment of psychosis in neurodegenerative disorders including Parkinson's disease, but the data remains equivocal and does not support its use. Only one agent-pimavanserin- is currently approved by the US Food and Drug Administration (FDA) for the treatment or neuropsychiatric symptoms in Parkinson's disease. This pharmacologic agent is approved for the treatment of hallucinations and delusions occurring in PD. Clinical trials with the goal of addressing neuropsychiatric symptoms without worsening other aspects of PD are currently limited. Since there is either little or no data concerning the use of psychotropics in patients with PD, clinicians are utilizing medication off-label.

Keywords: Parkinson's disease; Parkinson's Disease Dementia (PDD); Neurodegenerative Disorders (NDD); Neuropsychiatric Symptoms (NPS); Psychosis in Parkinson's Disease; Apathy in Parkinson's Disease; Fatigue in Parkinson's Disease; Impulse Control Disorders; Dopamine Dysregulation Syndrome 


\section{Introduction}

\section{Cognitive Impairment in Parkinson's Disease}

While PD can coexist with other common neurodegenerative or vascular diseases, dementia is increasingly recognized as common intrinsic features of PD [1]. Clinical features can generally distinguish between PD and other movement disorders associated with dementia, such as Dementia with Lewy Bodies (DLB) where cognitive impairment precedes motor parkinsonism by at least a year [2-4]. Cognitive impairment in PD, based on the extent to which it interferes with daily activities, it is generally divided into two major categories: Mild Cognitive Impairment (MCI) in the course of PD and PD Dementia (PDD) [1]. Both MCI and dementia are clinical diagnoses in patients with firmly established PD of at least one year's duration. Subtle cognitive impairment, particularly with frontal lobe features in the executive functions and planning domains, is frequently associated with PD and may appear early. These may also include less severe (though clear) memory difficulties and visuo-spatial difficulties [1]. Clinical criteria for the diagnosis MCI in patients with PD were first proposed by an international consensus panel in 2012 [5] and have since been validated. In studies of patients with a new diagnosis of $P D$, the prevalence of Mild Cognitive Impairment (MCI) ranges from approximately 10 to 35 percent [6].

Dementia should be suspected in patients with a decline in cognitive abilities that is interfering with daily function. The cognitive dysfunction of PDD is distinct from Alzheimer Disease (AD). With PDD, memory impairment is less prominent early on, while executive dysfunction and visual spatial impairments are features that may be apparent and functionally limiting before the patient meets criteria for dementia [1]. Dementia syndrome with insidious onset and slow progression, developing in the context of established PD is defined as: impairment in more than one cognitive domain (attention, executive function, visuospatial function, memory); decline from premorbid level, deficits severe enough to impair daily life (social, occupational, or personal care); independent of the impairment ascribable to motor or autonomic symptoms. Behavioral features such as apathy; changes in personality or mood, hallucinations, delusions, and Excessive Daytime Sleepiness (EDS) are supportive of the diagnosis but not required [7]. Cross-sectional studies suggest that the mean prevalence of dementia is 30 to 40 percent [8]. In prospective cohort studies, the incidence rate of dementia in patients with PD is consistently estimated at approximately 100 per 1000 patientyears, a rate almost five- to six fold higher than controls without PD [9]. It has been reported that approximately $30 \%$ of non-demented patients with PD developed dementia within 4 years; with almost $80 \%$ developing frank dementia after 8 years [10]. Cumulative incidence however rates of over 80 percent have been reported in patients followed for more than 20 years after onset of PD [11]. Dementia is thus not only common but nearly inevitable in patients with PD. Risk factors for developing PDD consist of: age at onset of $P D \geq 60$ years, duration of $P D$, and severity of parkinsonism [12]. Additional risk factors for the development of more severe or early cognitive impairment in patients with PD include the presence of rapid eye movement sleep behavior disorder (RBD), autonomic dysfunction, hyposmia, abnormal visual color discrimination, slow resting Electroencephalography (EEG) frequencies, and gait dysfunction [13]. Genetic may play a role in PD patients developing dementia. Majority of cases of PD appear to be sporadic and genetic risk factors for the development of dementia in those Patients had been suggested [8]. Genetic forms of PD often involve early or severe cognitive changes. Examples include PD associated with mutations in the Glucocerebrosidase (GBA) gene [14] and some forms of alphasynuclein (SNCA) associated PD [15]. Allelic variation in the gene encoding Microtubule-Associated Protein Tau (MAPT) appears to affect susceptibility as well $[16,17]$. Similarly, a high Catechol-0Methyl Transferase (COMT) activity haplotype has been found to be associated with a higher risk of developing cognitive impairment in PD [18]. By contrast, dementia is an uncommon feature of PD associated with parkin (PARK2) mutations [19].

Apolipoprotein E (APOE) gene polymorphism (well established as a risk factor for $\mathrm{AD}$ ) has been implicated as a potential risk for PDD. APOE $\varepsilon 4$ was found to be having greater effect on the severity other than an onset of dementia syndrome [20]. In a prospective cohort study the presence of the $\varepsilon 4$ allele was associated with rapid cognitive decline [17]. Cognitive impairment associated with overall decline in mental status could be complicated by psychosis. These are poor prognostic indicators and predict greater admission to nursing homes and early mortality. The presence of dementia and psychosis is as well the greatest limiting factor for the optimal use of anti-parkinsonian agents. The development of cognitive impairment in patients being treated for PD should prompt careful evaluation of the medical status of the patient and any underlying factors (systemic and focal infections, respiratory insufficiency, metabolic factors, changes to the environment and offending medications, such as sedatives and anticholinergic agents). Antiparkinsonian medications often require adjustment in this context as PD patients with cognitive decline are more sensitive to their side effects (i.e., anticholinergic agents; dopamine agonists). Every attempt should be made to simplify anti-PD medications by tapering off "adjunctive medications" (such as anticholinergics, MAO-B inhibitors, and amantadine; followed by dopamine agonists and COMT-inihibitors, if necessary) [21]. Ideally, PD patients with dementia should be on levodopa alone if at all possible [22].

Next step should include a trial of cholinesterase inhibitors and/or memantine in a stepwise fashion. Most, but not all, studies of cholinesterase inhibitors in PDD have noted a mild to moderate benefit $[23,24]$. Rivastigmine was evaluated in a 24-week, doubleblind, placebo-controlled study of 501 patients with mild to moderate PDD and was found to result in moderate improvement in dementia (as assessed by ADAS-cog) [25]. Metaanalysis points to improvement in about 15 percent of patients [26]. Donepezil 
also appeared to be associated with benefit by some primary and secondary outcome measures, including measures of executive function and attention. Another potential benefit of cholinesterase inhibitors in PDD is improvement in neuropsychiatric symptoms, such as hallucinations [26]. Treatment is continued if improvement is noted either on testing or by the family. Taper is warranted if there has been no improvement or if there are intolerable side effects. When cholinesterase inhibitors are discontinued, they should not be abruptly terminated, if at all possible, but rather tapered to avoid sudden cognitive and behavioral worsening [27]. Memantine could be a treatment option. Couple of trials with either Dementia with Lewy Bodies (DLB) or PDD found that patients treated with memantine performed better on the primary outcome assessment measure, the clinical global impression of change. Memantine was well tolerated in these trials $[28,29]$. Hallucinations and worsened neuropsychiatric symptoms however, have been anecdotally reported with the use of memantine PDD [30].

\section{Depression and Anxiety}

The incidence of depression and anxiety is greater in PD patients than in age-matched controls. It is the result of complex psychological and neurobiological factors [31]. The psychiatric burden doesn't solely come from functional decline associated with the threat of progressive motor dysfunction. Depression in PD follows a bi-modal distribution, with peaks of psychiatric burden around the time of onset of symptoms/diagnosis, as well as with loss of independence in late disease. There are several core and associated symptoms of depression (such as fatigue, apathy, sleep disruption, psychomotor retardation, weight loss) that are intrinsic to PD and confuse the assessment. Generalized anxiety disorder, panic disorder, social phobia, phobic disorder, agoraphobia, and obsessive compulsive disorder have all been described in PD $[23,24]$. Just like depression, anxiety can also be part of "pre-motor" manifestations of PD; and manifestation of wearing off phenomena. In such cases, management focuses primarily on levodopa dosing adjustments and other strategies to mitigate fluctuations. Clinicians should also be aware that akathisia occurs in patients with PD and should be differentiated from anxiety, which may cause similar clinical manifestations. Depression in patients with PD should be a target of focused therapy as studies have shown that depression in this population is a major determinant of quality of life. The modality of treatment should be tailored to the severity of the depressive symptoms. For mild depression associated with PD, nonpharmacological approaches may be most indicated. These include: supportive psychotherapy, and cognitive behavioral therapy [2,3]. CBT appears to be a particularly promising non-pharmacological approach [32] In more advanced depression pharmacotherapy is often indicated. Certain "off" phenomena, such as paroxysmal anxiety and panic may not respond well to antidepressant and anxiolytic therapy, but could respond to dopaminergic adjustments that minimize wearing off periods.
In terms of pharmacotherapy, experts recommend Dopamine Agonists (DA) as a first step in the management of depression of $\mathrm{PD}$ assuming it is compatible with the management of the motor symptoms of PD. Pramipexole (range of doses 0.3-4.2 mg/day) and ropinirole (10 mg/day) have antidepressant properties in patients with PD. Regular monitoring to capture symptoms like pathologic gambling, hypersexuality, and overspending in the course of DA therapy is however crucial [33]. Stronge vidence for the treatment of depression in PD has been reported with the Tricyclic Antidepressants (TCA's) [4]. However, these agents may be poorly tolerated due to anticholinergic effects and arrhythmogenic properties, particularly at higher doses. Selective Serotonin Reuptake Inhibitors (SSRI's) as well as Serotonin Noradrenaline Reuptake Inhibitors (SNRI's) may improve depressive and anxiety symptoms in patients with $\mathrm{PD}$, with minimal worsening of movement symptoms [34]. Where pharmacotherapy has been ineffective or poorly tolerated, or where depression in PD is severe, Electroconvulsive Therapy (ECT) and Transcranial Magnetic Stimulation (TMS) therapy may be helpful, though the evidence of efficacy for TMS in PD is still being gathered. It is important to mention that certain PD medications, such as dopamine agonists and MAO inhibitors, have demonstrated partial antidepressant effects in patients with PD, even when not being utilized for their pro-kinetic properties. These however are not typically used as sole treatment for management of depression in PD. There is a remarkable paucity of randomized clinical trials for the pharmacological management of anxiety in PD. However, based on clinical experience, the agents that are effective in the treatment of primary anxiety disorders (e.g. SSRIs), also appear to be effective in PD-related anxiety [31].

\section{Apathy}

Apathy can be defined as loss of or diminished motivation in comparison to the patient's previous level of functioning and which is not consistent with his age or culture. Symptoms in domains of behavior, cognition or and emotions should be consistently present for four weeks and cause clinically significant impairment in personal, social, occupational, or other important areas of functioning [35]. These changes in motivation may be reported by the patient himself or by the observations of othersJust as the symptoms of depression are sometimes hard to distinguish from those of PD itself (ie: psychomotor slowing, lack of motivation, bradykinesia), differentiating between depression, the symptoms of PD and apathy may also be challenging. Apathy appears to correlate well with greater severity of depression and functional impairment in PD and may be a predictor of dementia in the absence of depression [36]. There are no standardized treatments for apathy or abulia in the absence of depression or for persistent apathy despite treatment of depression. Antidepressants commonly used to treat depression in PD, such as SSRIs, are generally ineffective treatment options for apathy and abulia. Often, optimization of 
motor symptoms with dopamine agonists and levodopa to induce "on-states" may alleviate apathy to some degree. Psychostimulants such as amphetamine salts and methylphenidate may be effective, though response is often incomplete and variable. Bupropion may also show some benefit with minimal risk of worsening movement symptoms [24]. Based on results of a single small trial, the cholinesterase inhibitor rivastigmine may be considered, even in those without depression or cognitive dysfunction [37]. However, the clinical relevance and reproducibility of the changes observed in the trial are uncertain, and further studies are needed.

\section{Fatigue in PD- Assessment and Treatment}

Fatigue was proposed as one of the symptoms on Parkinson's spectrum. Differentiating between bradykinesia, sleepiness, apathy and fatigue may be difficult Suboptimally treated bradykinesia sometimes presents as subjective fatigue. However, in some cases fatigue appears in patients with mild bradykinesia and its origin is poorly understood. Treatment of fatigue in PD begins with an attempt to identify the cause. Excessive Daytime Sleepiness (EDS) and depression are both the most common and the most treatable identifiable causes. Potentially reversible causes, such as hypothyroidism and medication side effects, should be investigated as well [31]. Medications used for empiric treatment of fatigue, including amantadine and stimulants such as methylphenidate and pemoline, could be an option. However, the response to these is often insufficient. In one six weeks trial, 36 patients with PD were randomly assigned to treatment with either methylphenidate (10 mg three times daily) or placebo [36]. A significant reduction in two measures of self-reported fatigue for patients assigned to methylphenidate had been reported. Following this trial, a practice parameter from the American Academy of Neurology (AAN) concluded that methylphenidate is "possibly useful" for treating fatigue in patients with PD [38]. However, it is unclear whether the benefit of methylphenidate for fatigue in this setting is clinically meaningful. Of note, modafinil was not effective for improving either EDS (the primary outcome measure) or fatigue (a secondary outcome measure) in the randomized controlled trial [39].

\section{Psychosis In Parkinson's Disease}

Psychosis is broadly defined as a loss of contact with reality. It defines syndromes that impair both thought content and thought process which can result from any number of diseases and disorders. Disturbances in thought content include perceptions that are not based in reality, whereas disturbances in thought process reflect thoughts that are disorganized and illogical in form. Psychosis may present as acute or chronic, primary psychiatric or secondary in the course of other medical conditions [40]. Term psychosis envelopes two phenomena: delusions and hallucinations. Delusions are persistent beliefs that are not accepted in the context of a person's cultural and religious background, whereas hallucinations are false sensory perceptions. A slightly broader definition of psychosis can include disorganized speech or behavior. Symptoms of psychosis are typically episodic and these episodes are generally precipitated or exacerbated by psychosocial stressors $[1,2,40]$. Psychosis is debilitating symptom of $\mathrm{PD}$, it is an independent risk factor for nursing home placement, increases caregiver distress and patient mortality [41]. Consensus diagnostic criteria were established for psychosis in PD [42]. National Institute of Neurological Disorders and Stroke and the National Institute of Mental Health (NINDS$\mathrm{NIMH}$ ) criteria require the presence of at least one of the following: hallucinations, delusions, illusions, or false sense of presence. Psychosis must occur within the setting of a clear sensorium and a chronic course (> 1 month), either recurrent or continuous. Other medical, neurologic or psychiatric causes for the symptoms must be excluded [40].

The lifetime prevalence of psychosis in PD had been estimated for $50 \%$, with a range of $25 \%-60 \%$ depending on diagnostic criteria used $[43,44]$. Criteria for PD psychosis include then milder psychotic phenomena, such as visual illusions or sense of presence [40]. In one study of 116 PD patients, the cross-sectional prevalence of PD psychosis was $43 \%$ with the more traditional definition of psychosis, but with the more inclusive NINDS-NIMH criteria, increased to $60 \%[43,44]$. Visual hallucinations are the most common psychotic manifestation in PD. These hallucinations may be nonthreatening, brief, and well formed (reports of small children or animals are common). Hallucinations occur more frequently during times of decreased environmental stimulation, such as in the evening, low ambient lighting, or decreased sound levels. A false sense of presence or passage, as well as visual illusions, are considered minor psychotic phenomena but can affect anywhere from $17 \%-72 \%$ of PD patients [44]. While auditory, olfactory or tactile hallucinations are less common than their visual counterparts, they do occur; up to $20 \%$ of patients report auditory hallucinations. Nonvisual hallucinations or mixed hallucinations are more common in patients who develop psychotic symptoms at advanced ages [42] Minor hallucinations such as presence and passage hallucinations may precede the structured visual hallucinations [44].

Delusions occur in about 5\% of PD patients. Dopaminergic medications, cognitive dysfunction, depression, sleep disturbances, and longer duration of PD are risk factors for the emergence of psychosis in PD [45]. Tapering or elimination of antiparkinsonian medications, starting with the most recently added medications, is a first step, usually starting with those most likely to contribute to confusion. As mentioned before, if at all possible, psychotic PD patients should only be on levodopa for their motor symptoms [22]. Efforts to treat psychosis of PD with antipsychotics commonly used for primary psychotic disorders to date have been challenging and associated with significant deterioration of the motor symptoms [46]. Typical antipsychotics, particularly potent blockers of dopaminergic receptors, are usually contraindicated in PD. Observational studies which targeted PDD found beneficial effects of olanzapine in addressing psychosis especially delusions [46]. 
Worsening of motor function and overall psychiatric symptoms, however, has been reported in up to $80 \%$ of individuals with PDD following olanzapine treatment [47]. Risperidone was beneficial in managing psychotic symptoms of PDD. In the same group, treatment with risperidone improved levels of social, occupational, and psychological functioning. In practice, an unfavorable safety profile limits risperidone use [45]. Clozapine is efficacious in the treatment of PD psychosis as shown by randomized controlled trials even in relatively low dosing ranges (6.25 to $50 \mathrm{mg}$ daily) [48]. Clozapine is a selective D1 mesolimbic receptors blocker without significant binding to striatal dopamine D2 receptors. This selectivity together with its greater serotoninergic 5HT-2A/2C affinity, results in clozapine's favorable profile in managing PD psychosis. It is the antipsychotic medication that has consistently been found to be efficacious [45]. The risk of agranulocytosis and the necessity of blood monitoring with clozapine had led many experts to recommend a trial of other antipsychotics, mainly quetiapine (12.5-150 mg) before implementing use of clozapine Clozapine should be immediately stopped when the absolute neutrophil count is below $2000 \mathrm{~mm} 3$ and the white blood cell count is below $3.6 \mathrm{~mm} 3[46,48]$.

Quetiapine is the most frequently prescribed antipsychotic to target psychotic symptoms in PD. In its chemical structure (dibenzothiazepine) quetiapine is similar to clozapine. Findings are, however, inconsistent and firm conclusions about quetiapine efficacy cannot be drawn. One open label trial found a significant improvement in psychotic symptoms (as assessed by BPRS) in patients treated with quetiapine for 12 weeks. In this study the benefit of quetiapine (mean dose $91.5 \mathrm{mg} /$ daily) was comparable with a benefit of clozapine (mean dose $26 \mathrm{mg} /$ daily) [49]. A meta-analysis of data from different trials including a total of 241 participants randomized to either quetiapine or a comparator (placebo or clozapine) failed to show efficacy [50]. Several studies report improvement in the level of global clinical functioning of the PD patients with psychosis treated with quetiapine (as assessed by clinical global impression scales) $[49,50]$. Most studies indicate that patients taking quetiapine experienced fewer side effects compared to other antipsychotics; however, quetiapine has yet to be proven to be more effective than placebo in the treatment or psychosis in this population. In severe cases of PD psychosis, hospitalization should be considered in order to stabilize the patient. Somatic therapies may be an option in certain clinical situations. In PD, ECT was proved to be effective for depression and motor symptoms as well as for psychosis. It may be than considered to treat psychosis that has not responded to other interventions [51].

As noted earlier, the only pharmacological agent with FDA approval for treatment of PD psychosis is pimavanserin, a selectiveserotonin inverse agonist that preferentially targets 5-HT2A receptors, while avoiding activity at dopamine and other receptors commonly targeted by antipsychotics. The FDA's decision to approve pimavanserin was based on the results of a trial in which adults with PDP were randomly assigned to take $40 \mathrm{mg}$ of pimavanserin or placebo daily for six weeks. Patients taking pimavanserin experienced fewer and less severe hallucinations and delusions without motor side effects, which is commonly seen in other antipsychotics that function through dopamine blockade. The most common adverse effects reported by patients taking pimavanserin included peripheral edema, nausea, and confusion [52].

\section{Impulse Control Disorders and Dopamine Dysregulation Syndrome}

The Impulse Control Disorders (ICDs) are a family of neuropsychiatric conditions who central feature is the uncontrollable need to engage in repetitive behaviors, often to a maladaptive degree. Due to their intimate relationship to dopamine agonist and replacement therapy, impulse control disorders may represent a tremendous burden in and of themselves and may also limit optimal control of motor symptoms, leading to poorer functional outcomes and greater overall disease burden. The 4 most prominent treatment associated ICDs include compulsive gambling, hypersexuality, binge eating, and uncontrollable spending [40]. ICDs are found in almost $14 \%$ of patients being treated for PD, with pathological gambling and compulsive spending accounting for the largest percentage of cases [53]. ICDs are most prominent for patients taking dopamine agonists such as pramipexole and ropinirole, and may be enhanced when any of these are taken in conjunction with levodopa. Levodopa therapy alone is generally not associated with an increase in ICD's, except at very high dosages. Levodopa however is commonly associated with the related Dopamine Dysregulation Syndrome. Patients are often unlikely to volunteer the occurrence or increased urge to engage in impulsive behaviors such as hypersexuality and unrestrained gambling (though an attentive spouse of family member may bring it to the physician's attention). Certain ICDs may be idiosyncratic and specific to a particular lifelondtendencies (kleptomania, reckless generosity and hoarding), and therefore difficult to uncover with general screening. This may be particularly true with "hobby-ism" and other dopamine dysregulation disorders [53]. ICD in PD cluster with worse set-shifting and reward-related decision-making, and increased depression, anxiety, anhedonia, and impulsivity. All above have a negative impact on the quality of life of patients and their caregivers [54].

Patients with pre-existing or co-morbid impulse control disorders, as well as those with substance use disorders, Obsessive Compulsive Disorder (OCD) or tic disorders are at a greater risk for the development of treatment related ICDs and compulsive behaviors. Dopamine agonists should either be avoided in these patients if possible, or closely monitored to detect and avoid the occurrence of these disorders. In most patients, tapering and/or discontinuing dopamine agonist agents will improve or eliminate ICDs and compulsive behaviors. Generally, dopamine agonist 
therapy should be tapered slowly to reduce the risk of Dopamine Agonist Withdrawal Symptoms (DAWS) such as irritability, agitation, and dysphoria). Transition to levodopa is generally indicated in these situations. In regerds to symptomatic pharmacotherapy for ICD in PD patients -one randomized crossover trial of 17 patients found that amantadine (target dose $100 \mathrm{mg}$ twice daily), administered as add-on to baseline antiparkinsonian medications, and reduced pathologic gambling in all treated patients [55]. Limited evidence favores active treatment with naltrexone[56]. Dopamine Dysregulation Syndrome may require transition from levodopa to dopamine agonist [45]. Where dysregulated behaviors (impulsive, compulsive, and/or addictive tendencies) persist despite elimination of dopaminergic agents, there is little evidence available for management. Anecdotal reports have demonstrated variable benefit from atypical antipsychotics, naltrexone, and/ or mood stabilizers [57] Moreover, deep brain stimulation of the subthalamic nucleus might be a potential method in controlling impulsive and compulsive behaviors in Parkinson's disease [57-74].

\section{References}

1. Poletti M, Frosini D, Pagni C, Baldacci F, Nicoletti V, et al. (2012) Mild cognitive impairment and cognitive-motor relationships in newly diagnosed drug-naive patients with Parkinson's disease. J Neurol Neurosurg Psychiatry 83: 601-606.

2. Rothenberg KG (2017) Assessment and Management of Psychiatric Symptoms in Neurodegenerative Disorders (Chapter 23) In: NeuroGeriatrics (Edn) Tousi Babak and Cummings Jeffrey 2017, Springer International Publishing AG.

3. Rothenberg KG, Rajaram R (2019) Advances in Management of Psychosis in Neurodegenerative Diseases. Curr Treat Options Neurol Jan 21(1): 3.

4. Cummings J, Ritter A, Rothenberg KG (2019) Advances in Management of Neuropsychiatric Syndromes in Neurodegenerative Diseases Curr Psychiatry Rep 21(8): 79.

5. Litvan I, Goldman JG, Tröster AI, Schmand BA, Weintraub D, et al. (2012) Diagnostic criteria for mildcognitive impairment in Parkinson's disease: Movement Disorder Society Task Force guidelines. Mov Disord 27(3): 349-356.

6. Weintraub D, Simuni T, Caspell-Garcia C, Coffey C, Lasch S, et al. (2015) Cognitive performance and neuropsychiatric symptoms in early, untreated Parkinson's disease. Mov Disord 30: 919-927.

7. Emre M, Aarsland D, Brown R, Burn D, Duyckaerts C, et al. (2007) Clinical diagnostic criteria for dementia associated with Parkinson's disease. Mov Disord 22(12): 1689-1707.

8. Svenningsson P, Westman E, Ballard C, Aarsland D (2012) Cognitive impairment in patients with Parkinson's disease: Diagnosis, biomarkers, and treatment. Lancet Neurol 11(8): 697-707.

9. Aarsland D, Zaccai J, Brayne C (2005) A systematic review of prevalence studies of dementia in Parkinson's disease. Mov Disord 20(10): 12551263.

10. Buter TC, Van Den Hout A, Matthews FE, Larsen JP, Brayne C, et al. (2008) Dementia and survival in Parkinson disease: A 12-year population study. Neurology 70(13): 1017-1022.

11. Hely MA, Reid WG, Adena MA, Halliday GA, Morris GJ (2008) The Sydney multicenter study of Parkinson's disease: The inevitability of dementia at 20 years. Mov Disord 23(6): 837-844.

12. Levy G, Schupf N, Tang MX, Côté L, Louis E, et al. (2002) Combined effect of age and severity on the risk of dementia in Parkinson's disease. Ann Neurol 51(6): 722-729.
13. Anang JB, Gagnon JF, Bertrand JA, Romenets SR, Latreille V, et al. (2014) Predictors of dementia in Parkinson disease: A prospective cohort study. Neurology 83(14): 1253-1260.

14. Liu G, Boot B, Locascio JJ, Jansen IE, Winder-Rhodes S, et al. (2016) Specifically neuropathic Gaucher's mutations accelerate cognitive decline in Parkinson's. Ann Neurol 80(5): 674-685.

15. Obi T, Nishioka K, Ross OA, Terada T, Yamazaki K, et al. (2008) Clinicopathologic study of a SNCA gene duplication patient with Parkinson disease and dementia. Neurology 70(3): 238-241.

16. Williams-Gray CH, Evans JR, Goris A, Foltynie T, Ban M, et al. (2009) The distinct cognitive syndromes of Parkinson's disease: 5 year follow-up of the CamPaIGN cohort. Brain 132: 2958- 2969.

17. Morley JF, Xie SX, Hurtig HI, Stern MB, Colcher A, et al. (2012) Genetic influences on cognitive decline in Parkinson's disease. Mov Disord 27(4): 512-518.

18. Lin CH, Fan JY, Lin HI, Chang CW, Wu YR, et al. (2018) Catechol-Omethyltransferase (COMT) genetic variants are associated with cognitive decline in patients with Parkinson's disease. Parkinsonism Relat Disord 50: 48.-53.

19. Grünewald A, Kasten M, Ziegler A, Klein C (2013) Next-generation phenotyping using the parkin example: time to catch up with genetics. JAMA Neurol 70: 1186.

20. Fang L, Tang BS, Fan K, Wan CM, Yan XX, et al. (2018) Alzheimer's disease susceptibility genes modify the risk of Parkinson disease and Parkinson's disease-associated cognitive impairment. Neurosci Lett 677: 55-59.

21. Ehrt U, Broich K, Larsen JP, Ballard C, Aarsland D (2010) Use of drugs with anticholinergic effect and impact on cognition in Parkinson's disease: a cohort study. J Neurol Neurosurg Psychiatry 81(2): 160-165.

22. Hindle JV (2013) The practical management of cognitive impairment and psychosis in the older Parkinson's disease patient. J Neural Transm 120(4): 649-653.

23. Seppi K, Weintraub D, Coelho M, Perez-Lloret S, Fox SH, et al. (2011) The Movement Disorder Society Evidence-Based Medicine Review Update: Treatments for the non-motor symptoms of Parkinson's disease. Mov Disord 26 (Suppl 3): 42-80.

24. Connolly BS, Fox SH (2012) Drug treatments for the neuropsychiatric complications of Parkinson's Disease Expert Rev Neurother 12: 14391449.

25. Emre M, Aarsland D, Albanese A, Byrne EJ, Deuschl G, et al. (2004) Rivastigmine for dementia associated with Parkinson's disease. N Engl J Med 351(24): 2509-2518.

26. Maidment I, Fox C, Boustani M (2006) Cholinesterase inhibitors for Parkinson's disease dementia. Cochrane Database Syst Rev 25(1): CD004747.

27. Minett TS, Thomas A, Wilkinson LM, Daniel SL, Sanders J, et al. (2003) What happens when donepezil is suddenly withdrawn? An open label trial in dementia with Lewy bodies and Parkinson's disease with dementia. Int J Geriatr Psychiatry 18(11): 988-993.

28. Aarsland D, Ballard C, Walker Z, Bostrom F, Alves G, et al. (2009) Memantine in patients with Parkinson's disease dementia or dementia with Lewy bodies: a double-blind, placebo-controlled, multicentre trial. Lancet Neurol 8(7): 613-618.

29. Wang HF, Yu JT, Tang SW, Jiang T, Tan CC, et al. (2015) Efficacy and safety of cholinesterase inhibitors and memantine in cognitive impairment in Parkinson's disease, Parkinson's disease dementia, and dementia with Lewy bodies: systematic review with meta-analysis and trial sequential analysis. J Neurol Neurosurg Psychiatry 86(2): 135-143.

30. Monastero R, Camarda C, Pipia C, Camarda R (2007) Visual hallucinations and agitation in Alzheimer's disease due to memantine: report of three cases. J Neurol Neurosurg Psychiatry 78(5): 546. 
31. Weintraub D, Burn D (2011) Parkinson's Disease: The Quintessential Neuropsychiatric Disorder. Mov Disord 26(6): 1022-1031.

32. Troeung L, Egan SJ, Gasson N (2013) A meta-analysis of randomised placebo-controlled treatment trials for depression and anxiety in Parkinson's disease. PLoS One 8(11): e79510.

33. Barone P, Scarzella L, Marconi R, Antonini A, Morgante L, et al. (2006) Pramipexole versus sertraline in the treatment of depression in Parkinson's disease: a national multicenter parallel-group randomized study. J Neurol 253(5): 601-607.

34. Richard IH, Mc Dermott MP, Kurlan R, Lyness JM, Como PG, et al. (2012) A randomized, double-blind, placebo-controlled trial of antidepressants in Parkinson disease. Neurology 78(16): 1229-1236.

35. Mulin E, Leone E, Dujardin K, Delliaux M, Leentjens A, et al. (2011) Diagnostic criteria for apathy in clinical practice. Int J Geriatr Psychiatry 26(2): 158-165.

36. Dujardin K, Sockeel P, Delliaux M, Destée A, Defebvre L (2009) Apathy May Herald Cognitive Decline and Dementia in Parkinson's Disease Mov Disord 24(16): 2391-2397.

37. Devos D, Moreau C, Maltête D, Lefaucheur R, Kreisler A, et al. (2014) Rivastigmine in apathetic but dementia anddepression-free patients with Parkinson's disease: A double-blind,placebo-controlled, randomised clinical trial. J Neurol Neurosurg Psychiatry 85(6): 668-674.

38. Zesiewicz TA, Sullivan KL, Arnulf I, Chaudhuri KR, Morgan JC, et al. (2010) Practice Parameter: treatment of nonmotor symptoms of Parkinson disease: report of the Quality Standards Subcommittee of the American Academy of Neurology. Neurology 74(11): 924-931.

39. Ondo WG, Fayle R, Atassi F, Jankovic J (2005) Modafinil for daytime somnolence in Parkinson's disease: double blind, placebo controlled parallel trial. J Neurol Neurosurg Psychiatry 76(12): 1636-1639.

40. (2013) American Psychiatric Association Diagnostic and statistical manual of mental disorders ( $5^{\text {th }}$ edn.). Arlington, VA: APA.

41. Marsh L, Williams JR, Rocco M, Grill S, Munro C, et al. (2004) Psychiatric comorbidities in patients with Parkinson disease and psychosis. Neurology 63(2): 293-300.

42. Ravina B, Marder K, Fernandez HH, Friedman JH, Mc Donald W, et al. (2007) Diagnostic criteria for psychosis in Parkinson's disease: report of an NINDS, NIMH work group. Mov Disord 22(8): 1061-1068.

43. Fenelon G, Soulas T, Zenasni F, De Langavant LC (2010) The changing face of Parkinson's disease-associated psychosis: a cross-sectional study based on the new NINDS-NIMH criteria Mov Disord 25(6): 755-759.

44. Fenelon G, Alves G (2010) Epidemiology of psychosis in Parkinson's disease. J Neurol Sci 289: 12-17.

45. Goetz CG (2010) New Developments in Depression, Anxiety, Compulsiveness, and Hallucinations in Parkinson's disease. Mov Disorders 25(Suppl 1): 104-109.

46. Goldman JG, Holden S (2014) Treatment of psychosis and dementia in Parkinson's disease. Curr Treat Options Neurol 16(3): 281-299.

47. Moretti R, Torre P, Antonello RM, Cazzato G, Griggio S, et al. (2003) Olanzapine as a treatment of neuropsychiatric disorders of Alzheimer's disease and other dementias: A 24-month follow-up of 68 patients. Am J Alzheimers Dis Other Demen18(4): 205-214.

48. Pollak P, Tison F, Rascol O, Destée A, Péré JJ, et al. (2004) Clozapine in drug induced psychosis in Parkinson's disease: a randomised, placebo controlled study with open follow up. J Neurol Neurosurg Psychiatry 75(5): 689-695.

49. Morgante L, Epifanio A, Spina E, Zappia M, Di Rosa AE, et al. (2004) Quetiapine and clozapine in parkinsonian patients with dopaminergic psychosis. Clin Neuropharmacol 27(4): 153-156.

50. Desmarais P, Massoud F, Filion J, Nguyen QD, Bajsarowicz P (2016) Quetiapine for Psychosis in Parkinson Disease and Neurodegenerative
Parkinsonian Disorders: A Systematic Review. J Geriatr Psychiatry Neurol 29(4): 227-236.

51. Hausner L, Damian M, Sartorius A, Frolich L (2011) Efficacy and cognitive side effects of ECT in depressed elderly inpatients with co-existing mild cognitive impairment or dementia. J Clin Psychiatry 72: 91-97.

52. Cummings J, Isaacson S, Mills R, Williams H, Chi-Burris K, et al. (2014) Pimavanserin for patients with Parkinson's disease psychosis: a randomised, placebo-controlled phase 3 trial Lancet 383(9916): 533540 .

53. Weintraub D, Claassen DO (2017) Impulse Control and Related Disorders in Parkinson's disease. Int Rev Neurobiol 133: 679-717.

54. Martini A, Dal Lago D, Edelstyn NMJ, Grange JA, Tamburin S (2018) Impulse Control Disorder in Parkinson's Disease: A Meta-Analysis of Cognitive, affective, and Motivational Correlates. Front Neurol 9: 654.

55. Thomas A, Bonanni L, Gambi F, Di Iorio A, Onofrj M (2010) Pathological gambling in Parkinson disease is reduced by amantadine. Ann Neurol 68(3): 400-404.

56. Papay K, Xie SX, Stern M, Hurtig H, Siderowf A, et al. (2014) Naltrexone for impulse control disorders in Parkinson disease: a placebo-controlled study. Neurology 83(9): 826-833.

57. Zhang G, Zhang Z, Liu L, Yang J, Huang J, et al. (2014) Impulsive and compulsive behaviors in Parkinson's disease. Front Aging Neurosci 6: 318.

58. Gallagher DA, Schrag A (2012) Psychosis, apathy, depression and anxiety in Parkinson's disease. Neurobiology of Disease 46(3): 581-589.

59. Barone P, Antonini A, Colosimo C, Marconi R, Morgante L, et al. (2009) The PRIAMO study: A multicenter assessment of nonmotor symptoms and their impact on quality of life in Parkinson's disease Mov Disord 24(11): 1641-1649.

60. Lin YQ, Chen SD (2018) RBD: A red flag for cognitive impairment in Parkinson's disease? Sleep Med 44: 38-44.

61. Blonder LX, Slevin JT (2011) Emotional Dysfunction in Parkinson's Disease. Behav Neurol 24(3): 201-217.

62. Stoyka LE, Arrant AE, Thrasher DR, Russell DL, Freire J, et al. (2020) Behavioral defects associated with amygdala and cortical dysfunction in mice with seeded $\alpha$-synuclein inclusions. Neurobiol Dis 134: 104708.

63. Bohnen NI, Kaufer DI, Hendrickson R, Ivanco LS, Lopresti BJ, et al. (2006) Cognitive correlates of cortical cholinergic denervation in Parkinson's disease and parkinsonian dementia. J Neurol 253(2): 242-247.

64. Shimada H, Hirano S, Shinotoh H, Aotsuka A, Sato K, et al. (2009) Mapping of brain acetylcholinesterase alterations in Lewy body disease by PET. Neurology 73(4): 273-278.

65. Dubois B, Tolosa E, Katzenschlager R, Emre M, Lees AJ, et al. (2012) Donepezil in Parkinson's disease dementia: a randomized, double-blind efficacy and safety study. Mov Disord 27(10): 1230-1238.

66. Ekman U, Eriksson J, Forsgren L, Mo SJ, Riklund K, et al. (2012) Functional brain activity and presynaptic dopamine uptake in patients with Parkinson's disease and mild cognitive impairment: A crosssectional study. Lancet Neurol 11(8): 679-687.

67. Marini P, Ramat S, Ginestroni A, Paganini M (2003) Deficit of short-term memory in newly diagnosed untreated parkinsonian patients: reversal after L-dopa therapy. Neurol Sci 24(3): 184-185.

68. Morrison CE, Borod JC, Brin MF, Hälbig TD, Olanow CW (2004) Effects of levodopa on cognitive functioning in moderate-to-severe Parkinson's disease (MSPD). J Neural Transm (Vienna) 111: 1333-1341.

69. Bohnen NI, Albin RL, Müller ML, Petrou M, Kotagal V, et al. (2015) Frequency of cholinergic and caudate nucleus dopaminergic deficits across the predemented cognitive spectrum of Parkinson disease and evidence of interaction effects. JAMA Neurol 72(2): 194-200. 
70. Murai T, Muller U, Werheid K, Sorger D, Reuter M, et al. (2001) In vivo evidence for differential association of striatal dopamine and midbrainserotonin systems with neuropsychiatric symptoms in Parkinson's disease. J Neuropsychiatry Clin Neurosci 13(2): 222-228.

71. Huot P, Johnston TH, Darr T, Hazrati LN, Visanji NP, et al. (2010) Increased 5-HT2A receptors in the temporal cortex of parkinsonian patients with visual hallucinations. Mov Disord 25(10): 1399-408.

72. Ballanger B, Strafella AP, Van Eimeren T, Zurowski M, Rusjan PM, et al. (2010) Serotonin 2A receptors and visual hallucinations in Parkinson disease. Arch Neurol 67(4): 416-421.

ISSN: 2574-1241

DOI: 10.26717/BJSTR.2020.28.004713

Kasia Gustaw Rothenberg. Biomed J Sci \& Tech Res

(C) This work is licensed under Creative

Submission Link: https://biomedres.us/submit-manuscript.php
73. Rothenberg KG, Wiechers IR (2015) Antipsychotics for Neuropsychiatric Symptoms of Dementia-Safety and Efficacy in the Context of Informed Consent. Psychiatric Annals 45(7): 348-353.

74. Mendonça DA, Menezes K, Jog MS (2007) Methylphenidate improves fatigue scores in Parkinson disease: a randomized controlled trial. Mov Disord 22(14): 2070-2076.

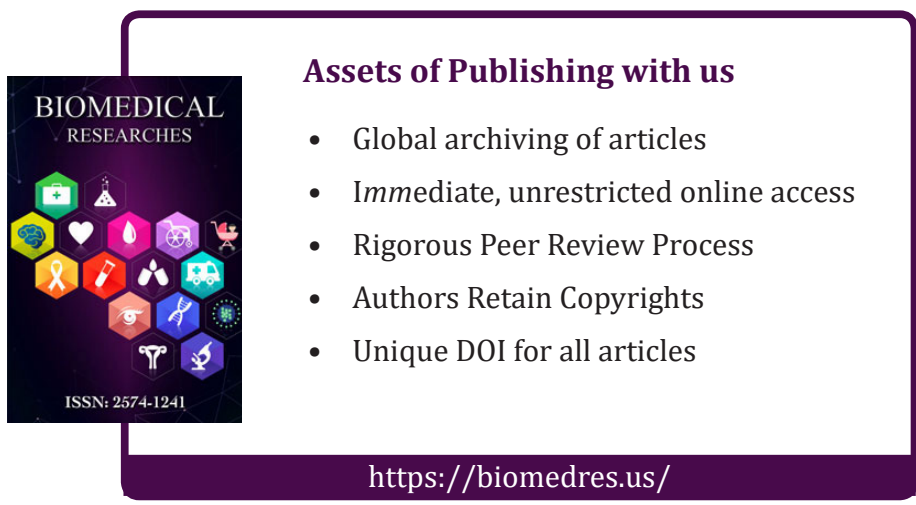

